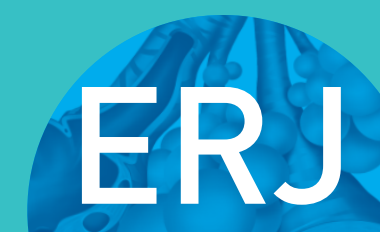

open research
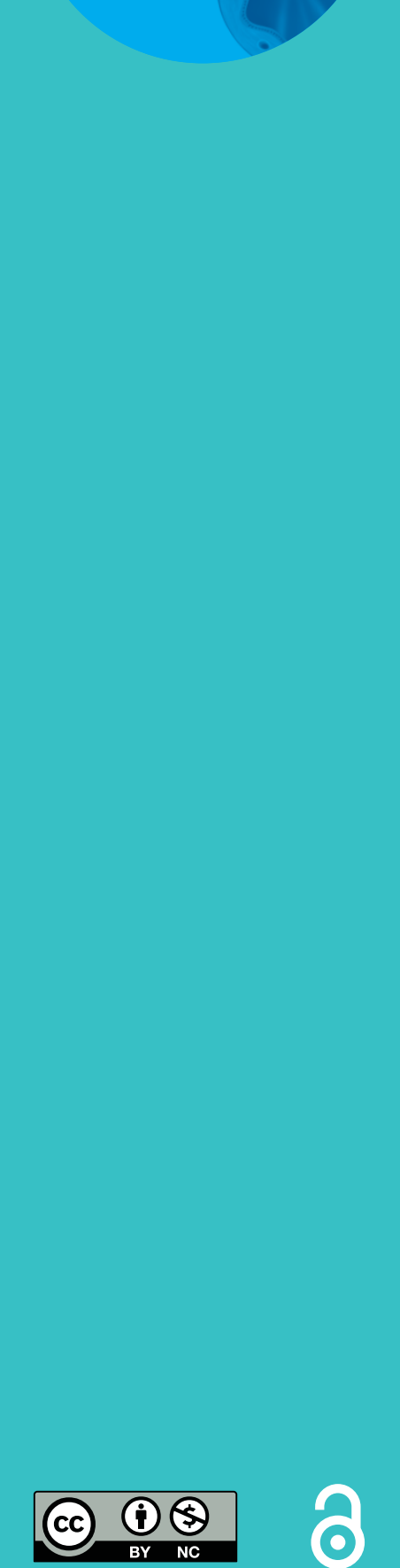

\section{Mortality rates for chronic lower respiratory diseases in Italy from 1979 to 2010: an age-period-cohort analysis}

\section{Giancarlo Pesce}

Affiliation: Unit of Epidemiology and Medical Statistics, Dept of Diagnostics and Public Health, University of Verona, Verona, Italy.

Correspondence: Giancarlo Pesce, Università di Verona c/o Istituti Biologici II, Strada Le Grazie 8, 37134 Verona, VR, Italy. E-mail: giancarlo.pescedunivr.it

ABSTRACT Chronic lower respiratory diseases (CLRDs) are a major cause of morbidity and mortality worldwide. The objectives of this study were to estimate the trends in CLRD mortality in Italy, and the specific contributions of age, time period and birth cohort in driving these trends.

Population and cause-of-death data in Italy between 1979 and 2010 were collected from the World Health Organization website. Age-specific mortality rates for CLRDs, and effects for age, time period and birth cohort on mortality trends were estimated using age-period-cohort models.

Chronic obstructive pulmonary disease (COPD) and chronic bronchitis represent nearly $98 \%$ of the deaths from CLRDs. Despite the overall number of deaths have been stable (in men) or increasing (in women), the age-standardised rates have been steadily decreasing from 1979 to 2010, passing from 104.3 to 55.4 per 100000 person-years in men and from 32.2 to 19.6 per 100000 person-years in women. The average relative annual decrease was $-3.6 \%$ in men and $-2.7 \%$ in women. Since the end of the $1990 \mathrm{~s}$, the decreasing trend of CLRD mortality has started to level off, in particular in women. The decrease in CLRD mortality rates has been more accentuated in more recent cohorts and in younger age groups.

Both birth cohort and time period significantly affected the CLRD mortality rates, suggesting that changes in the spread of risk factors (smoking habits, early-life and occupational exposures) across different birth cohorts, as well as in advanced in healthcare and medical practice, may have played a major role in secular changes in COPD mortality rates in Italy.

@ERSpublications

COPD ASRs have dropped in the last 30 years in Italy but number of deaths is stable in men and increasing in women http://ow.ly/Y19oD

This article has supplementary material available from openres.ersjournals.com

Received: Dec 032015 | Accepted after revision: Feb 042016

Conflict of interest: None declared

Copyright $\odot$ ERS 2016. This article is open access and distributed under the terms of the Creative Commons Attribution Non-Commercial Licence 4.0. 


\section{Introduction}

Chronic lower respiratory diseases (CLRDs) are a group of pathologies that obstruct the lungs, which mainly includes chronic obstructive pulmonary disease (COPD) and asthma. CLRDs are an important cause of morbidity and mortality; the most deadly is COPD, which represents the third cause of death worldwide [1]. The prevalence of COPD increases rapidly with age, and its morbidity and mortality are particularly significant in the elderly [2]. In Italy, about one out of four people aged 65-84 years suffers from COPD or asthma, which coexist in a substantial proportion of subjects [3].

Italy is one of the countries with longest life expectancy in the world, and CLRDs have a great social and economic impact in Italy [4, 5]. In 2012, CLRDs caused about 22000 deaths in Italy (3.6\% of the total), ranking as the fourth cause of death, after cardiovascular diseases, tumours of the airways and Alzheimer disease [6].

The study of the temporal trends of CLRD mortality, and of how they relate to time period, birth cohort and age of the subjects, may help to better understand the aetiology of the disease, and to address clinical and public health strategies. In this work, the trends in CLRD mortality between 1979 and 2010 in Italy were estimated, analysing the specific contributions of age, period and cohort in driving these trends.

\section{Methods}

\section{Data sources}

Mortality data on chronic lower respiratory diseases (CLRDs) and population data for the period 1979-2010 in Italy were obtained from the World Health Organization (WHO) cause-of-death query online application (http://apps.who.int/healthinfo/statistics/mortality/causeofdeath_query/start.php). For each calendar year, data were reported by sex and age, tabulated in 5-year age groups and specific underlying cause of death, defined as "the disease or injury which initiated the train of morbid events leading directly to death" in accordance with the rules of the International Classification of Diseases (ICD). Three different ICD versions have been used to classify the causes of death during that time period: the ICD Ninth Revision (ICD-9) basic tabulation list from 1979 to 1995 , the ICD-9 detailed full list from 1996 to 2002 and the ICD-10 detailed full list from 2003 to 2010. The specific ICD codes included in this study are shown in table 1. Mortality data for 2004 and 2005 were not present in the database, while data recorded from 1951 to 1978, which had been coded using the ICD-7 and ICD-8 (tabulation list), were not included in this study because of the impossibility of isolating specifically the deaths due to CLRDs from other respiratory diseases.

\section{Data analysis}

Approximate birth cohorts were estimated by subtracting the midpoint of the 5-year age group from the corresponding calendar year.

\section{TABLE 1 Causes of death for chronic lower respiratory diseases, with corresponding}

International Classification of Diseases (ICD) codes

$\begin{array}{ll}\text { ICD-9 basic tabulation list (from } 1979 \text { to } \mathbf{1 9 9 5} \text { ) } \\ \text { B323 } & \text { Bronchitis, chronic and unspecified, emphysema, and asthma } \\ \text { B324 } & \text { Bronchiectasis } \\ \text { B325 } & \text { Other chronic obstructive pulmonary diseases } \\ \text { ICD-9 detailed full list (from } 1996 \text { to 2002) } \\ 490 & \text { Bronchitis, not specified as acute or chronic } \\ 491 & \text { Chronic bronchitis } \\ 492 & \text { Emphysema } \\ 493 & \text { Asthma } \\ 494 & \text { Bronchiectasis } \\ 495 & \text { Extrinsic allergic alveolitis } \\ 496 & \text { Chronic airway obstruction, not elsewhere classified } \\ \text { ICD-10 detailed full list (from } 2003 \text { to } 2010 \text { ) } \\ \text { J40 } \\ \text { J41 } & \text { Bronchitis, not specified as acute or chronic } \\ \text { J42 } & \text { Simple and mucopurulent chronic bronchitis } \\ \text { J43 } & \text { Unspecified chronic bronchitis } \\ \text { J44 } & \text { Emphysema } \\ \text { J45 } & \text { Other chronic obstructive pulmonary disease } \\ \text { J46 } & \text { Asthma } \\ \text { J47 } & \text { Status asthmaticus } \\ \end{array}$


Incidence rates, reported by sex and age group, were calculated by dividing the total number of deaths for each cause by the population at risk in the equivalent time period. Age-standardised mortality rates (ASRs) per 100000 person-years in males and females for each year from 1979 to 2010 were calculated using the 2013 European Standard Population as reference [7].

As deaths for CLRDs were extremely rare in young ages, and since the over-85 population was grouped together for some of the considered years in the WHO's cause-of-death query online application, the age-period-cohort (APC) analyses were restricted to the population aged 35-84 years.

Temporal trends of mortality are driven by the merged effects of age, time period and birth cohort. In the present study, an APC analysis, based on a Poisson regression model, was performed to disentangle the independent effects of age, cohort and period. Because of the exact link between the three variables (e.g. the age at death can be calculated directly by the difference between the date of death and the date of birth), the APC models suffer from the so-called identifiability problem. Thus, to obtain identifiable answers for each of the temporal parameters, the models need to be constrained to ensure that three functions showing the age, time period and birth cohort effects can be identified [8].

Using this approach, a "drift" parameter, which represents the sum of the overall linear time trend for time period and birth cohort effects, was extracted. Deviations from linearity (curvature), which can be uniquely attributable to cohort or period effects and are not dependent on any model constraint, are then interpreted as specific cohort and period effects $[9,10]$.

The birth cohort and time period effects were displayed as rate ratios (RR). The last time period available in our data (2010) was set as a reference period and the drift parameter was included in the time period effect, making the age effect interpretable as age-specific rates in the 2010 after adjustment for cohort effects. The cohort effect function was set to 1 on average with 0 slope, and is interpretable as the cohort-related RR after adjustment for age and period [8].

Age, time period and birth cohort estimates were modelled by natural cubic spline functions, with five knots at subsequent quantiles for age, period and cohort [8]. APC effects were calculated separately for males and females, and age-specific male-to-female ratios were derived from age effects after adjusting for birth cohort and time period effects.

Data analyses were performed using STATA 13.1 (StatCorp, College Station, TX, USA) and the Epi package in R 3.1 .1 (www.r-project.org) [8, 11].

\section{Results}

Specific cause of death from CLRDs

Between 2006 and 2010, the main cause of mortality from CLRDs was COPD/emphysema (ICD-10 codes J43-J44), which accounted about $78.6 \%$ and $84.8 \%$ of the total CLRD deaths in women and men, respectively, followed by chronic bronchitis ( J40-J42) (figure 1). Asthma ( J45-J46) was responsible of about 3.7\% of CLRD deaths in women and $1.5 \%$ in men, while bronchiectasis ( J47) represented $<1 \%$ of the total deaths for CLRDs.

\section{Trends in mortality from CLRDs}

From 1979 to 2010, CLRDs had been the cause of death of about 20000 people per year in Italy and about two out of three of them were men (table $1 \mathrm{~S}$ in the supplementary material). The crude mortality rates
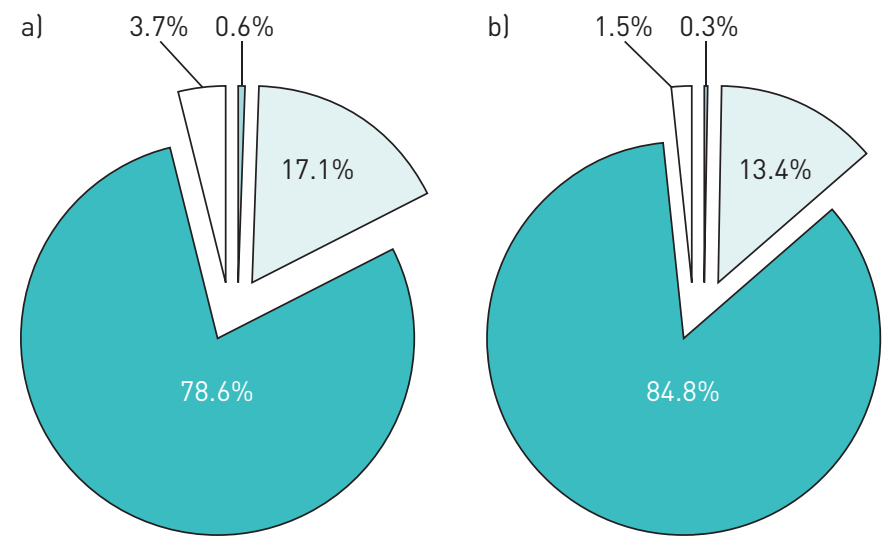

$\square$ Chronic bronchitis (J40-J42)

FIGURE 1 Specific causes of death for chronic lower respiratory diseases in Italy, 2006-2010. a) Females; b) males. COPD: chronic obstructive pulmonary disease. 
remained almost unchanged in men, passing from 46.2 per 100000 person-years in 1979 to 47.4 in 2010 , while they increased from 18.7 to $27.0(+44 \%)$ in women (figure 2$)$. However, in the same period, the ASRs dropped from 32.2 to 19.6 per 100000 person-years in women, and, more noticeably, from 104.3 to 55.4 per 100000 person-years in men.

Figure 3 summarises the crude age-specific mortality rates in men and women, stratified by calendar year and birth cohort. In all birth cohorts and time periods, the mortality rates increased exponentially with age, and visibly decreased in more recent birth cohorts and time periods. In relative terms, the mortality rates decreased more softly in the most elderly group ages; for example, the rates declined by $-38 \%$ in the 80-84-year age group (from 454 to 282 per 100000 person-years) compared to a drop of $-78 \%$ in the 60-64-year age group (from 48.5 to 10.8 per 100000 person-years) (table 2).

\section{APC effects on mortality from CLRDs}

The independent effects of age (plotted as mortality rates relative to the year 2010), birth cohort and time period (plotted as RRs) were evaluated through APC analysis and are illustrated in figure 4.

The analysis of deviance for the APC regression models indicated that the linear time trend (drift) was the most relevant determinant to explain the variation over time of asthma incidence rates (table $2 S$ ). Moreover, birth cohort and time period significantly contributed to deviations from linearity (curvatures) in the trends of mortality.

Overall, during the period 1979-2010, the rates of mortality averagely decreased by $-3.6 \%$ per year in men and $-2.7 \%$ per year in women (weighted drift) (figure 4). This indicates that, in the 30 years between 1980 and 2010, the mortality rates decreased by about three-fold in men and two-fold in women. In both men and women, the deeper decrease was observed between 1985 and the second half of the 1990s, when the rates underwent a substantial stabilisation, especially in women, interrupting the otherwise linear decrease in mortality trends. After adjusting for age and period, the mortality risk for CLRDs was lowest in subjects born in more recent cohorts compared to those born in the 1920s, who had the highest risk. This suggests that, while mortality rates decreased across all ages, the rates decreased primarily in younger subjects. For example, it can be observed that between 1980 and 2010 the mortality rates decreased three- to four-fold in subjects aged 40 years, but less than two-fold for subjects aged 80 years.

The age-specific mortality rates relative to the year 2010 are also illustrated in figure 4 . In both men and women, the rates increased exponentially with age and they are always greater in men compared to women. The male-to-female RR significantly varies with age, men having a two-fold greater risk of dying of CLRDs than women at 40 years old, and up to 3.5-fold at the age of 70 years (figure 5).

\section{Discussion}

The main results of the analyses are as follows.

1) COPD (including emphysema and chronic bronchitis) is the main contributor of CLRD mortality in Italy, causing about $97 \%$ of total CLRD deaths, while asthma accounts for about $2 \%$ of CLRD deaths.

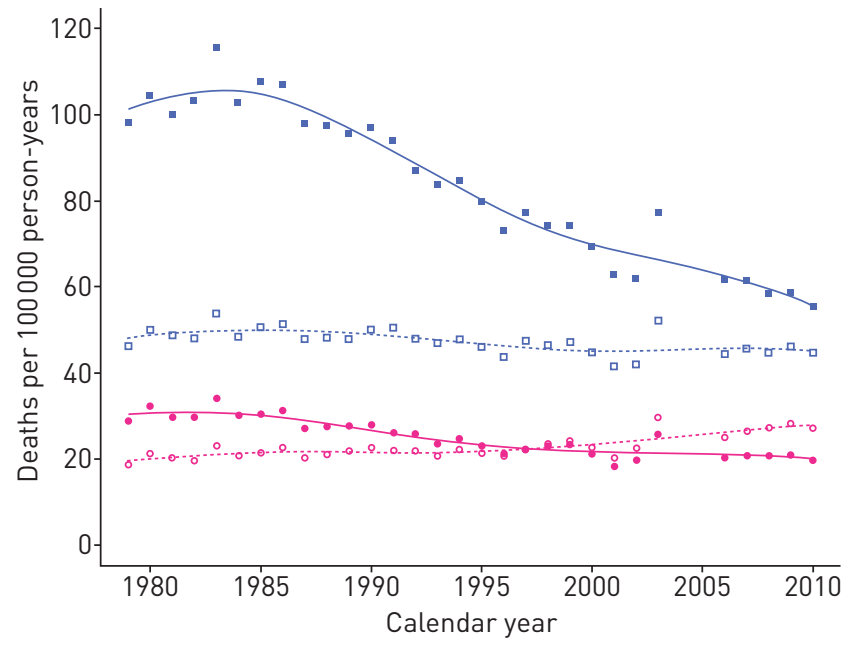

FIGURE 2 Crude and age-standardised mortality rates for chronic lower respiratory diseases in Italy, 1979-2010. Open and filled symbols represent the crude and age-standardised rates, respectively; blue squares and pink circles indicate rates in men and in women, respectively. 

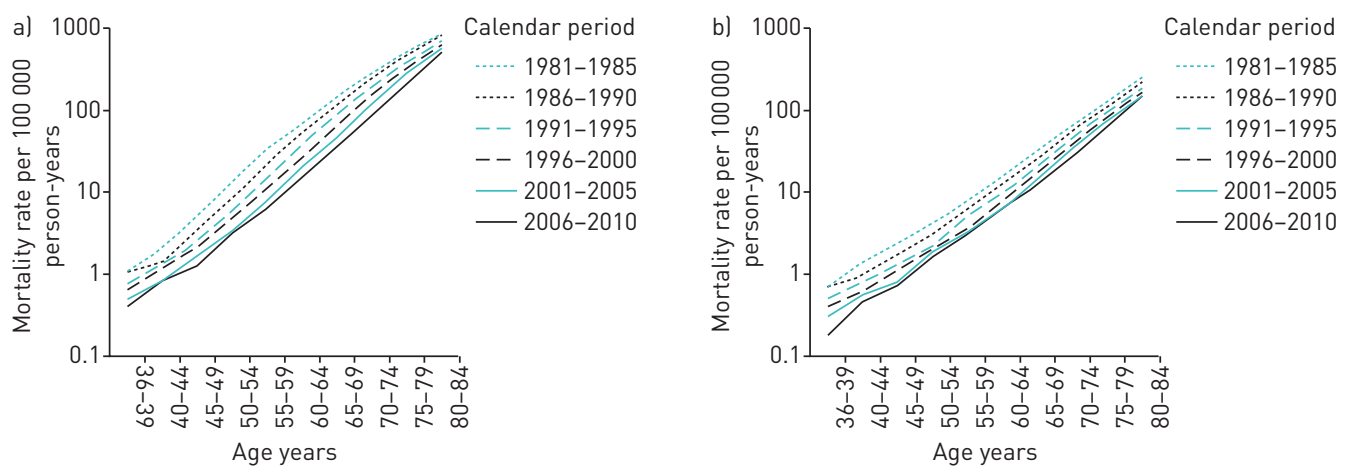

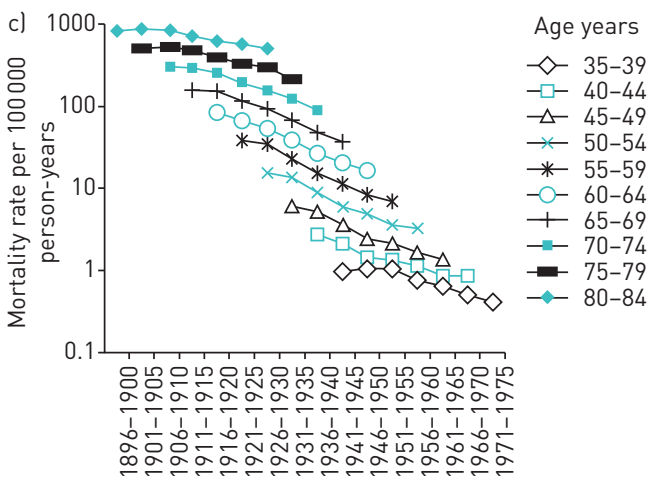

Birth cohort

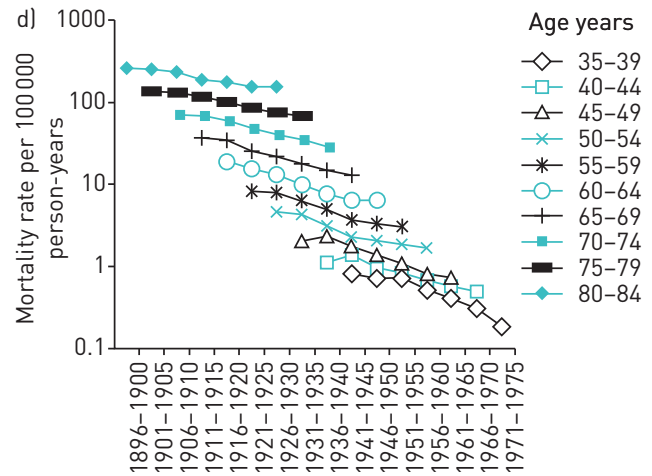

Birth cohort

FIGURE 3 Age-specific mortality rates of chronic lower respiratory diseases by time period of diagnosis in a) men and b) women, and birth cohort in c) men and d) women.

TABLE 2 Mortality rates of chronic lower respiratory diseases (per 100000 person-years) by age at onset and time period in Italy

\begin{tabular}{|c|c|c|c|c|c|c|c|c|}
\hline \multirow{2}{*}{$\begin{array}{l}\text { Age } \\
\text { years }\end{array}$} & \multicolumn{7}{|c|}{ Calendar period } & \multirow[t]{2}{*}{ Total } \\
\hline & $1979-1980$ & $1981-1985$ & $1986-1990$ & 1991-1995 & $1996-2000$ & $2001-2005^{\#}$ & $2006-2010$ & \\
\hline $0-4$ & 0.7 & 0.6 & 0.6 & 0.5 & 0.3 & 0.1 & 0.0 & 0.4 \\
\hline $5-9$ & 0.2 & 0.2 & 0.2 & 0.2 & 0.1 & 0.0 & 0.0 & 0.1 \\
\hline $10-14$ & 0.2 & 0.3 & 0.3 & 0.2 & 0.2 & 0.0 & 0.1 & 0.2 \\
\hline $15-20$ & 0.4 & 0.3 & 0.4 & 0.4 & 0.3 & 0.1 & 0.1 & 0.3 \\
\hline $20-24$ & 0.3 & 0.4 & 0.3 & 0.4 & 0.3 & 0.2 & 0.1 & 0.3 \\
\hline $25-29$ & 0.3 & 0.4 & 0.4 & 0.4 & 0.3 & 0.3 & 0.1 & 0.3 \\
\hline $30-34$ & 0.4 & 0.6 & 0.6 & 0.5 & 0.4 & 0.3 & 0.2 & 0.4 \\
\hline 35-39 & 0.9 & 0.9 & 0.9 & 0.6 & 0.5 & 0.4 & 0.3 & 0.6 \\
\hline $40-44$ & 1.9 & 1.7 & 1.2 & 1.0 & 0.9 & 0.7 & 0.6 & 1.1 \\
\hline $45-49$ & 4.0 & 3.7 & 2.6 & 1.9 & 1.6 & 1.2 & 1.0 & 2.2 \\
\hline 50-54 & 9.8 & 8.8 & 5.8 & 4.0 & 3.4 & 2.6 & 2.3 & 4.9 \\
\hline 55-59 & 21.6 & 20.3 & 13.8 & 9.9 & 7.2 & 5.6 & 4.8 & 11.2 \\
\hline $60-64$ & 48.5 & 39.9 & 31.9 & 23.2 & 16.7 & 13.3 & 10.8 & 23.8 \\
\hline 65-69 & 90.2 & 84.8 & 64.6 & 53.9 & 40.2 & 29.8 & 23.9 & 51.1 \\
\hline $70-74$ & 166.2 & 160.1 & 139.1 & 109.3 & 89.8 & 73.1 & 55.2 & 105.9 \\
\hline $75-79$ & 278.6 & 283.7 & 253.6 & 215.4 & 180.2 & 159.2 & 127.8 & 202.0 \\
\hline $80-84$ & 454.6 & 463.0 & 439.6 & 374.3 & 329.1 & 300.4 & 282.4 & 358.7 \\
\hline$>85$ & 653.2 & 727.4 & 741.1 & 705.6 & 663.4 & 673.1 & 665.9 & 686.2 \\
\hline Total & 33.7 & 35.1 & 35.0 & 34.3 & 33.9 & 34.4 & 35.7 & 34.7 \\
\hline ASR & 57.7 & 58.7 & 53.9 & 46.8 & 40.7 & 37.6 & 34.2 & 45.2 \\
\hline
\end{tabular}




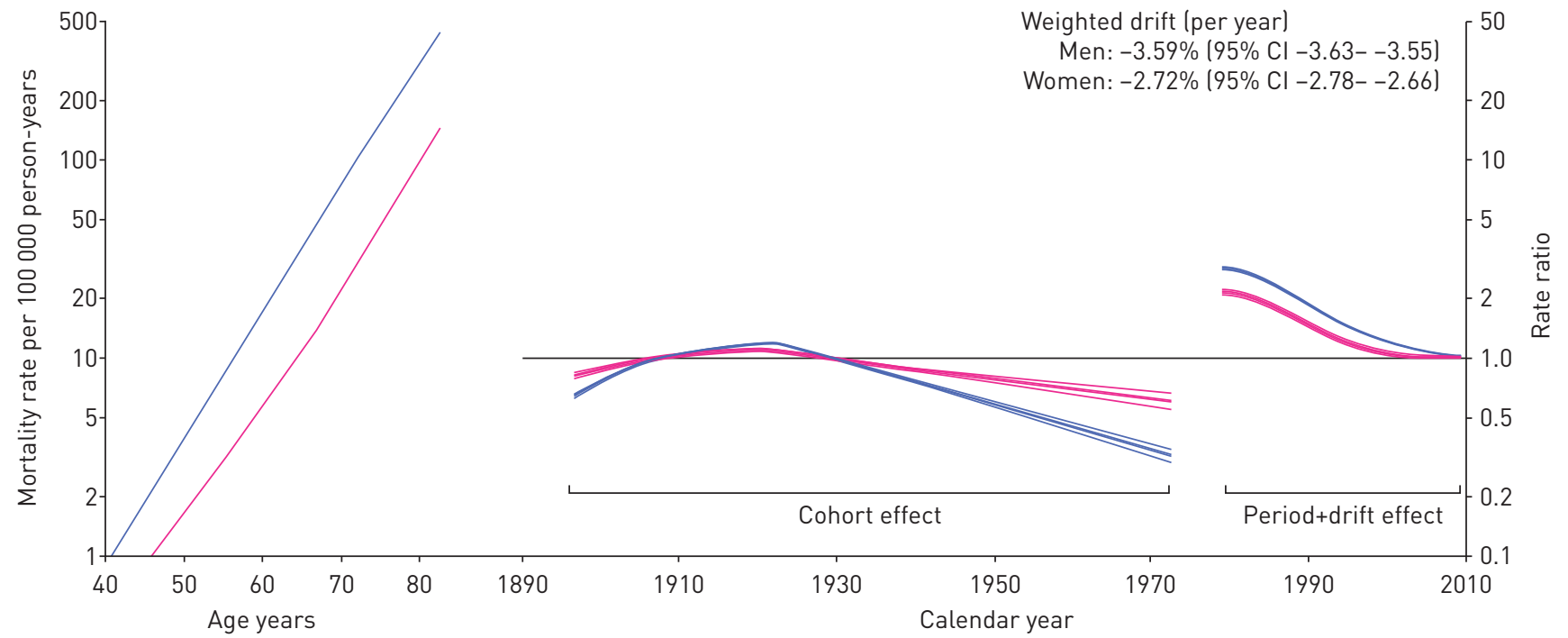

FIGURE 4 Age, cohort and time period effects and weighted drift of mortality rates for chronic lower respiratory diseases for men (blue lines) and women (pink lines) in Italy, 1978-2010. Age effects are presented as mortality rates for the reference time period (2010); time period effects are presented as rate ratios relative to 2010; and cohort effects are constrained to be 1, on average, with 0 slope. All effects are modelled using cubic splines.

2) Despite the overall number of deaths from CLRDs have been stable (in men) or increasing (in women), the age-adjusted CLRD mortality rates have been steadily decreasing from 1980 to 2010 .

3) From 1980 to 2010 , the average relative annual decrease in mortality rates was $-3.6 \%$ in men and $-2.7 \%$ in women; however, since the end of the 1990s, the decreasing trend of CLRD mortality has started to level off, in particular in women.

4) The decrease in CLRD mortality rates has been more accentuated in more recent cohorts and in younger age groups.

In this study, we analysed the trends in mortality from CLRDs, which pool together the deaths caused by a group of different pathologies: COPD (including emphysema and bronchitis), asthma and bronchiectasis. The use of a broad definition such as CLRDs as an outcome is deliberate, as it helped to compare data collected from 1979 to 2010, through different ICD revisions (e.g. asthma, emphysema and bronchitis have the same code in ICD-9 basic tabulation, while they are separate in the detailed full ICD-9 and ICD-10 lists), and to reduce issues of misclassification. In fact, it is not easy for physicians to make differential diagnosis between these diseases because they are strongly related, share common symptomatology and risk factors, and tend to overlap, especially in the most severe cases. Asthma-COPD overlap syndrome (ACOS) affects a substantial part of the population, which is more likely to be admitted to hospital and to have respiratory symptoms and physical impairment when compared to asthma or COPD alone [3].

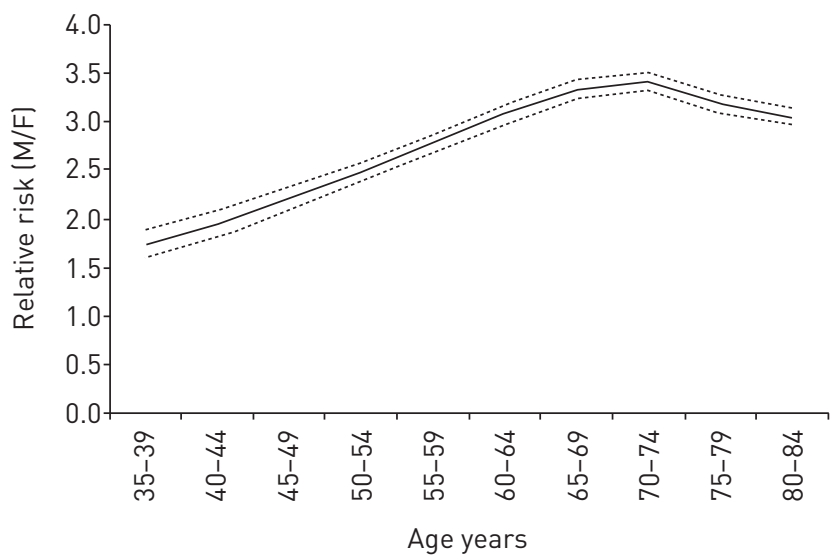

FIGURE 5 Male-to-female (M/F) ratios of mortality for chronic lower respiratory diseases by age group according to the age-period-cohort model in Italy, 1978-2010. Dashed lines represent 95\% confidence intervals. 
Looking at the specific ICD codes, $97.3 \%$ of deaths from CLRDs were provoked by COPD (including emphysema and chronic bronchitis (ICD-10 J40-J44)) and $0.4 \%$ by bronchiectasis (J47), while asthma (J45-J46) accounted for the remaining $2.3 \%$ of deaths. Therefore, it can be realistically assumed that the trends in CLRD mortality observed in this study are almost exclusively driven by COPD.

Recent studies have highlighted that, despite that the ASRs of COPD have fallen significantly worldwide in the last 20 years, COPD has passed from the fourth to the third most common cause of death at global level [1]. In agreement with this, our results show that the total number of deaths from COPD in Italy remained stable in men and even increased in women, despite the age-adjusted rates of COPD mortality significantly decreasing in the last 30 years. Similar trends were shared by other European Union (EU) countries and Japan [12,13]. As noted by BuRNey et al. [1], the apparent paradox of the decreasing ASRs and increasing numbers of death can be easily explained by the modification in the age structure of the population. In fact, life expectancy has improved in Italy, and the proportion of elderly people, who have greater mortality rates for COPD, has considerably increased in the last 30 years. For example, the population aged $>65$ years in Italy passed from $13 \%$ to $20 \%$ of the total population $(+50 \%)$, and the population aged $>80$ years passed from $2.2 \%$ to $5.9 \%(+160 \%)$. Given that the ASRs in mortality have begun to level off, while the ageing of the Italian population is still ongoing, the number of deaths from COPD will probably not diminish in the next decades. Moreover, as COPD mortality has declined less than mortality from other causes, the proportion of people dying of COPD is likely to grow in forthcoming years.

The temporal trends of COPD mortality are driven by the combined effects of age, time period and birth cohort effects. These effects are proxies of underlying secular changes that occurred in Italy, including changes in demographic factors, lifestyles and healthcare. In particular, the age effect provides information on the different rates of mortality across different age groups; cohort effects are longitudinal, as they highlight possible differences in the spread of risk factors, such as smoking habits or healthier lifestyles, across different birth cohorts. Conversely, time period effects are cross-sectional, reflecting changes in screening practices, diagnostic techniques or disease classification that involve all age groups simultaneously.

In the period between 1978 and 2010, the average relative annual decrease (drift) in age-adjusted mortality rates has been $-3.6 \%$ and $-2.7 \%$ in men and women, respectively; this means that in the last 30 years, the rates have dropped of nearly three- and two-fold, respectively, in men and in women.

Smoking is the most important and modifiable aetiological factor for the development of COPD. About $50 \%$ of lifelong smokers develop COPD, compared with only $10 \%$ of never-smokers [14]. The prevalence of lung function abnormalities and of respiratory symptoms is higher in smokers, who suffer from a faster decline of forced expiratory volume in $1 \mathrm{~s}$ than nonsmokers. The consumption and the prevalence of cigarette smoking steadily increased, especially in women, reaching a peak in the late 1970s, when they progressively started to decrease $[15,16]$. The prevalence of current tobacco smoking in the Italian population aged 14 years and over dropped from 35\% to $23 \%$ from 1980 to 2010 (figure 6). This trend was mainly driven by men, among whom the prevalence of current smokers fell from $54 \%$ to $29 \%$, while the prevalence remained stable at $17 \%$ in women during the same period [17]. The reduction in smoking initiation and consumption, especially in men, is likely to be a major contributor to the fall in mortality rates in more recent birth cohorts [13]. Moreover, the greater attention payed in recent years to the damage from second-hand smoking might also have contributed to the decrease in COPD exacerbations and mortality in nonsmokers [18].

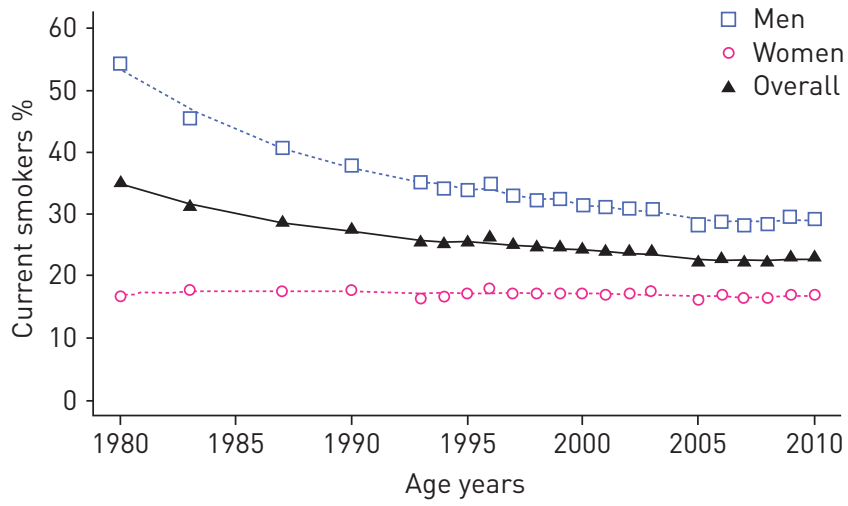

FIGURE 6 Prevalence of tobacco smoking among Italian population (aged 14 years and over), 1980-2010. Data from [17]. 
Independently from cigarette smoking, occupational exposures are thought to be responsible of about $15-20 \%$ of all COPD cases [19]. The social-economic transition of Italy, with fewer people occupied in at-risk jobs such as mining, agriculture, or the textile and chemical industries, and the improvement in work safety policies may have reduced, especially in men, the exposure to airborne pollutants, thus contributing to the remarkable reduction in COPD mortality observed in the last 30 years.

Viral and bacterial infections are the most common cause of COPD exacerbations, and accelerate the decline in lung function that characterises COPD [20]. The prevention of infections through the increasing spread of vaccination campaigns for seasonal influenza and colds in the elderly population, and the improvement of healthcare and hygienic conditions both in households and hospitals may have greatly contributed to a reduction in COPD mortality.

Moreover, evidence suggests an important role in early-life factors in the development of COPD in later life, and some researchers have suggested that the proportion of the risk of COPD attributable to early-life environmental factors may be as great as that attributable to smoking [21]. It has been reported that airway function is significantly diminished in adults born with low birthweight or who had respiratory infections in infancy $[21,22]$. Improvements in perinatal nutritional and hygienic conditions in cohorts born more recently may have influenced the long-term function of the lungs in the offspring, reducing their risk of developing CLRDs in adult age.

COPD mortality rates increase exponentially with age, reflecting the accumulation of exposures with passing years, the physiological deterioration of the lung function and the increased vulnerability to pathogens given by the weakening of the immune system. The rates of mortality were considerably higher in men than women. One of the reasons for this difference might be the different occupational exposure to noxious agents and the higher prevalence of cigarette smoking in men. Recent reports highlight that the differences in smoking habits between men and women has been continuously reducing in recent decades in Italy [17]. This figure might explain, at least in part, why the drop in COPD mortality has been more striking in men than women in the last 30 years. The "gender equalisation" in smoking habits will be also likely to influence future trends in COPD morbidity: in fact, women may become more susceptible than men to developing COPD when exposed to risk factors such as tobacco smoking, reducing the differential of COPD mortality between the sexes.

As previously mentioned, the trends in age-standardised mortality in men and women reported in Italy are similar in other EU countries, such as Germany, Belgium and Spain [13]. This suggests that APC effects observed in Italy might be similar to those of other countries, even if generalisation from Italy to other EU countries must be done with extreme caution, considering that EU countries have quite different demographic, sociological and economic profiles.

A limitation of this study is that trends were not further controlled for other potential confounding factors, because of the absence of data for relative age groups and time periods.

This study used the WHO cause of death query online application, which includes complete aggregate data about the underlying cause of deaths from 117 countries. The underlying causes of death were defined as "the disease or injury which initiated the train of morbid events leading directly to death" and were codified according to the standardised ICD. The underlying causes of deaths data are potentially biased by incomplete records. Moreover, as deaths often result from a complex interaction between multiple diseases and factors, the process of coding underlying causes of death involves some extent of misattribution or miscoding because it can be difficult, even for medically qualified staff, to identify a single underlying cause that originated the aetiological chain leading to death. A recent publication showed that it is likely that the real burden of COPD-related deaths is underestimated when evaluated based only on the underlying cause of death from death certificates [23].

\section{Conclusions}

Despite that ASRs for COPD have been dropping considerably in the last 30 years in Italy, the number of deaths for COPD is stable in men and even increasing in women. This paradox can be explained by changes in the age structure of the Italian population. Both birth cohort and time period significantly affected the COPD mortality rates, suggesting that changes in the spread of risk factors across different birth cohorts, as well as in advanced in healthcare and medical practice, may have played a major role in secular changes in COPD mortality rates in Italy.

\section{References}

1 Burney PGJ, Patel J, Newson R, et al. Global and regional trends in COPD mortality, 1990-2010. Eur Respir J 2015; 45: 1239-1247.

2 Buist S, McBurnie MA, Vollmer WM, et al. International variation in the prevalence of COPD (the BOLD study): a population-based prevalence study. Lancet 2007; 370: 741-750. 
3 de Marco R, Pesce G, Marcon A, et al. The coexistence of asthma and chronic obstrictive pulmonary disease (COPD): prevalence and risk factors in young, middle-aged and elderly people from the general population. PLoS One 2013; 8: e62985.

4 Pauwels RA, Rabe KF. Burden and clinical features of chronic obstructive pulmonary disease (COPD). Lancet 2005; 364: 613-620.

5 Accordini S, Corsico AG, Calciano L, et al. The impact of asthma, chronic bronchitis and allergic rhinitis on all-cause hospitalizations and limitations in daily activities: a population-based observational study. BMC Pulm Med 2015; 15: 10.

6 Italian Institute of Statistics. Le principali cause di morte in Italia [The principal causes of death in Italy]. www.istat.it/it/archivio/140871 Date last accessed: January, 2016.

7 Pace M, Lanzieri G, Glickman M, et al. Revision of the European Standard Population: report of Eurostat's task force. Eurostat: methodologies and working papers. Luxembourg, Publications Office of the European Union, 2013.

8 Carstensen B. Age-period-cohort models for the Lexis diagram. Statist Med 2007; 26: 3018-3045.

9 Clayton D, Schifflers E. Models for temporal variation in cancer rates. II: Age-period-cohort models. Stat in Med 1987; 6: 469-481.

10 Holford TR. Approaches to fitting age-period-cohort models with unequal intervals. Biometrics 1983; 39: 311-324.

11 Rutherford MJ, Lambert PC, Thompson JR. Age-period-cohort modeling. Stata J 2010; 10: 606-627.

12 Lopez-Campos JL, Ruiz-Ramos M, Soriano JB. Mortality trends in chronic obstructive pulmonary disease in Europe, 1994-2010: a joinpoint regression analysis. Lancet Respir Med 2014; 2: 54-62.

13 Pham TM, Ozasa K, Kubo T, et al. Age-period-cohort analysis of chronic obstructive pulmonary disease mortality in Japan, 1950-2004. J Epidemiol 2012; 22: 302-307.

14 Lundbäck B, Lindberg A, Lindström M, et al. Not 15 but 50\% of smokers develop COPD? Report from the Obstructive Lung Disease in Northern Sweden studies. Respir Med 2003; 97: 115-122.

15 Sardu C, Mereu A, Pitzalis G, et al. Smoking trends in Italy from 1950 to 2000. J Epidemiol Community Health 2006; 60: 799-803

16 Verlato G, Melotti R, Corsico AG, et al. Time trends in smoking habits among Italian young adults. Respir Med 2006; 100: 2197-2206.

17 Italian Institute of Statistics. Fumatori di 14 anni e più per ripartizione geografica e sesso - Anni 1980, 1983, 1987, 1990, 1993-2013 [Smoking in age 14 years and older by geographical division and sex - years 1980, 1983, 1987, 1990, 1993-2013]. http://seriestoriche.istat.it/index.php?id=7\&user_100ind_pi1\%5Bid_pagina\%5D=33\&cHash= 6490cf56b560420d1bd686e863104bea Date last accessed: January, 2016.

18 Oberg M, Jaakkkola MS, Woodward A, et al. Worldwide burden of disease from exposure to second-hand smoke: a retrospective analysis of data from 192 Countries. Lancet 2011; 377: 139-146.

19 Balmes J, Becklake M, Blanc P, et al. Occupational contribution to the burden of airway disease. Am J Respir Crit Care Med 2003; 167: 787-797.

20 Wedzicha JA, Donaldson GC. Exacerbations of Chronic Obstructive Pulmonary Disease. Respir Care 2003; 48: 1204-1215.

21 Svanes C, Sunyer J, Plana E, et al. Early life origins of chronic obstructive pulmonary disease. Thorax 2010; 65: $14-20$.

22 Barker DJ, Godfrey KM, Fall C, et al. Relation of birth weight and childhood respiratory infection to adult lung function and death from chronic obstructive airway disease. BMJ 1991; 303: 671-675.

23 Marcon A, Saugo M, Fedeli U. COPD-related mortality and co-morbidities in northern Italy, 2008-2012: a multiple causes of death analysis. COPD 2015; 13: 1-7. 